\title{
Enhancing High-Order Harmonic Generation in Light Molecules by Using Chirped Pulses
}

\author{
M. Lara-Astiaso, ${ }^{1}$ R. E. F. Silva, ${ }^{1}$ A. Gubaydullin, ${ }^{1}$ P. Rivière, ${ }^{1}$ C. Meier, ${ }^{2}$ and F. Martín ${ }^{1,3,4, *}$ \\ ${ }^{1}$ Departamento de Química, Universidad Autónoma de Madrid, 28049 Madrid, Spain \\ ${ }^{2}$ Laboratoire de Collisions Agrégats Réactivité, IRSAMC, UMR CNRS 5589, Université Paul Sabatier, \\ 31062 Toulouse, France \\ ${ }^{3}$ Instituto Madrileño de Estudios Avanzados en Nanociencia, 28049 Madrid, Spain \\ ${ }^{4}$ Condensed Matter Physics Center (IFIMAC), Universidad Autónoma de Madrid, 28049 Madrid, Spain
}

(Received 20 January 2016; published 24 August 2016)

\begin{abstract}
One of the current challenges in high-harmonic generation is to extend the harmonic cutoff to increasingly high energies while maintaining or even increasing the efficiency of the high-harmonic emission. Here we show that the combined effect of down-chirped pulses and nuclear dynamics in light molecules allows one to achieve this goal, provided that long enough IR pulses are used to allow the nuclei to move well outside the Franck-Condon region. We also show that, by varying the duration of the chirped pulse or by performing isotopic substitution while keeping the pulse duration constant, one can control the extension of the harmonic plateau.
\end{abstract}

DOI: 10.1103/PhysRevLett.117.093003

The interaction of intense infrared laser pulses with atomic or molecular gases gives rise to emission of short, subfemtosecond bursts of UV and x-ray light every halfcycle of the driving laser. This process, known as highharmonic generation (HHG), was discovered in the late 1980s [1-3] and subsequently explained by theory [4-6], initiating a new discipline known as attophysics $[7,8]$. HHG is nowadays well established as a powerful source of ultrashort coherent light in the extreme ultraviolet (XUV) and soft $\mathrm{x}$-ray range, with the inherent advantage to be realizable on tabletop devices. The typical harmonic emission spectrum consists of a series of peaks corresponding to odd multiples of the driving laser frequency and exhibits a plateau region followed by a sharp cutoff at an energy approximately given by [4]

$$
E_{\text {cutoff }}=I_{p}+3.17 I_{0} \lambda^{2} / 4,
$$

where $I_{p}$ is the ionization potential of the atomic system, $I_{0}$ is the laser peak intensity, and $\lambda$ is the laser wavelength. XUV and $\mathrm{x}$-ray attosecond pulses have permitted us to time resolve ultrafast physical processes such as Auger decay following inner shell photoionization [9] or electron tunneling [10], as well as, within pump-probe schemes, to provide unprecedented insights into electron [11-14] and, more recently, coupled electron-nuclear dynamics [15-17] in atoms and molecules, respectively.

In the past decade, a considerable effort has been devoted to face two challenges of high-harmonic generation. The first one is to extend the harmonic cutoff to even higher energies $[18,19]$, thus opening the way to study diffraction effects in molecules (e.g., X-ray or photoelectron diffraction) or electron dynamics resulting from $K$-shell ionization. The other one is to maintain or even increase the efficiency of the conversion process at such high-harmonic energies [20], so that the modest yields achieved in current time-resolved experiments increase accordingly. Extending the cutoff energy can, in principle, be achieved, according to Eq. (1), by increasing either the laser peak intensity $I_{0}$ [21] or the wavelength $\lambda$ (e.g., into the far IR region) [22-24], while increasing the harmonic yield can be trivially achieved by increasing the peak intensity of the laser. However, the latter is limited by saturation of the ionization yield $[25,26]$, which occurs when the ground state of the irradiated system is fully depleted. Another complication is that the harmonic yield decreases with the wavelength of the driving field according to the approximate power law $\lambda^{-5} \sim \lambda^{-6}[27,28]$, so that extending the cutoff is usually accompanied by a decrease of the harmonic emission.

Consequently, more elaborate schemes have been proposed to extend the cutoff region, such as the use of two delayed pulses [29], color mixing techniques [30,31], spatial inhomogeneities [32,33], a combination of the above [34], or XUV-assisted HHG [35]. Some of them $[30,32]$ have already been realized in the lab, but their widespread implementation is somewhat limited due to their intrinsic complexity (use of several pulses, synchronization, preexistence of attosecond pulses, etc). Another alternative is the use of intense few-cycle chirped pulses, which have been proposed for atoms [36-38] and diatomic molecules [39] but with rather limited success.

Recent theoretical work on HHG from diatomic molecules containing light nuclei has shown that, in addition to modulations due to the multicenter nature of the molecular potential [40], nuclear motion can significantly alter HHG emission and, possibly, the above analysis. For example, Lein et al. [41,42] have shown that the harmonic cutoff is significantly reduced in $\mathrm{H}_{2}^{+}$compared to that in $\mathrm{D}_{2}^{+}$and $\mathrm{T}_{2}^{+}$, a phenomenon that is entirely due to nuclear dynamics. Nuclear wave packet dynamics in $\mathrm{H}_{2}^{+}$is also responsible for the redshift of harmonic peaks [43] and for emission of 
even harmonics due to electron localization $[44,45]$. Furthermore, the HHG spectrum has also been shown to depend on the initial vibrational state $[46,47]$.

In this Letter, we show that the combined effect of nuclear dynamics and pulse frequency chirp allows us to considerably extend the harmonic cutoff of the $\mathrm{H}_{2}^{+}$molecule while keeping or even increasing the harmonic yield. The effect is observed only for down chirps and for IR pulses that are long enough to induce nuclear wave packet dynamics able to bring the molecule well outside the Franck-Condon (FC) region. We also show that, by varying the pulse duration, typically between 10 and 20 IR cycles, or the mass of the nuclei, one can control the extension of the cutoff.

We consider a linearly polarized IR field and a collinear model in which the molecule is aligned with that field. Rotational effects are ignored. These approximations are justified by the large intensity of the field, the shortness of the IR pulses, and the slowness of rotational motion in comparison with electronic and vibrational motions. In this framework, we have solved the time-dependent Schrödinger equation (TDSE) in full 3D dimensionality by using cylindrical coordinates $(\rho, z, R)$, where the first two coordinates define the electron position and $R$ is the internuclear distance [48]. Notice that we have taken advantage of the cylindrical symmetry of the problem to remove the azimuthal coordinate $\phi$. Since the details of this 3D model have been already published [49], here we only describe its main ingredients. The TDSE for $\mathrm{H}_{2}^{+}$is given by (atomic units are used throughout unless otherwise stated)

$$
\left[H_{\mathrm{el}}(\rho, z, R)+T(R)+V(z, t)-i \frac{\partial}{\partial t}\right] \Psi(\rho, z, R, t)=0,
$$

where $H_{\mathrm{el}}=T_{\mathrm{el}}+V_{\mathrm{en}}+1 / R$ is the electronic time-independent Hamiltonian, $T(R)$ the nuclear kinetic energy operator, and $V(z, t)=z E(t)$ the external potential created by the laser field, in the length gauge. Likewise, $T_{\mathrm{el}}$ and $V_{\mathrm{en}}$ stand for the electron kinetic energy operator and the electron-nuclei interaction, respectively. The external laser field is given by the expression $E(t)=f(t) \cos \left(\omega_{0} t+\right.$ $\left.\frac{1}{2} \alpha t^{2}+\delta_{0}\right)$, where $\omega_{0}$ is the central frequency and $f(t)$ the pulse envelope, defined as $f(t)=E_{0} \cos ^{2}(\pi t / T)$ for $|t| \leq T / 2$. The envelope is characterized by the total pulse duration $T$ and the field amplitude $E_{0}$, related to the peak intensity $I_{0}=c E_{0}^{2} /(8 \pi)$. Throughout the Letter, we have considered pulses with $I_{0}=3 \times 10^{14} \mathrm{~W} / \mathrm{cm}^{2}$ and $\lambda=800 \mathrm{~nm}$. These pulses have a frequency $\omega(t)=\omega_{0}+$ $\alpha t$ (calculated as the time derivative of the total phase [50]) that depends on time, and thus we say that the pulse is upchirped if $\alpha>0$ or down-chirped if $\alpha<0$. The carrierenvelope phase is always set to $\delta_{0}=0$, since the conclusions of our study are independent of that phase.

The TDSE calculations have been carried out in a nonequidistant cubic grid defined within the boundaries of the numerical box, $|z|<160,|\rho|<30$, and $|R|<15$ a.u. The grid spacings correspond to $\Delta \rho=0.075, \Delta z=0.1$, and $\Delta R=0.05$ a.u. at the center of the box and increase gradually towards the outer grid boundaries. Wave function absorbers are used for $|z|>150,|\rho|>25$, and $|R|>15$ a.u. to avoid spurious reflections. For the time propagation, we solve the TDSE in the Crank-Nicolson scheme with a splitoperator method. The time step used for the electronic motion is $\Delta t_{\mathrm{el}}=0.011$ a.u. and for the nuclear propagation $\Delta t_{\mathrm{el}}=0.11$ a.u., which is performed only every ten electronic time steps. The convergence of the whole set of parameters has been checked. At each time step, we compute the dipole response in the acceleration form: $\ddot{d}(t)=-\langle\Psi(\rho, z, R, t)|\mathcal{O}(z, t)| \Psi(\rho, z, R, t)\rangle$, where $\mathcal{O}(z, t)=$ $-\nabla V(z, t)+E(t)$ is the dipole acceleration operator. The harmonic spectrum is given by the square of the Fourier transform of $\ddot{d}(t)$.

Figure 1 shows the harmonic spectra obtained for 5-, 10-, and 15-cycle pulses, with and without chirp. For all durations, both chirp signs lead to the same spectral broadening. For the 5-cycle pulse, the results obtained with chirped and unchirped fields are qualitatively similar, including the harmonic cutoff, which according to Eq. (1) is
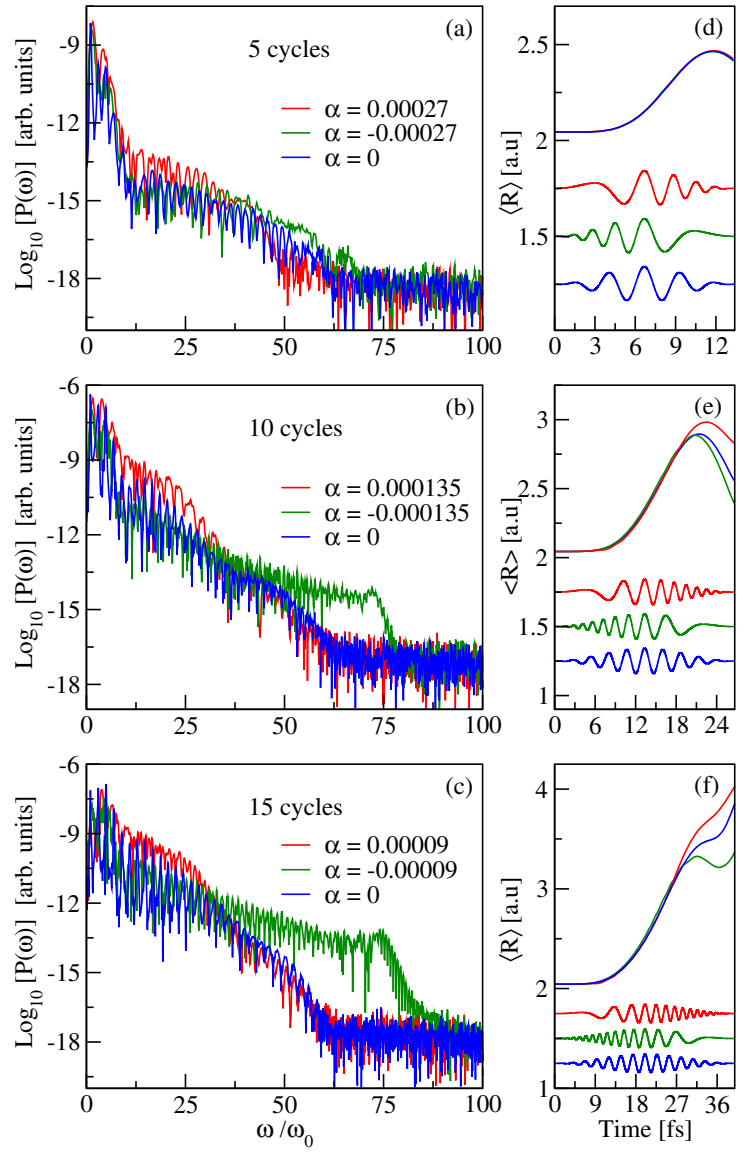

FIG. 1. High-harmonic spectra of $\mathrm{H}_{2}^{+}$obtained with chirped and unchirped pulses of (a) 5, (b) 10, and (c) 15 cycles. The chirp coefficient $\alpha$ changes proportionally to the pulse duration to keep invariant the spectrum of chirped pulses. (d)-(f) display the pulses used to obtain the corresponding harmonic spectra as well as the mean values of the internuclear distance $\langle R\rangle$ as functions of time $t$. The origin of $t$ has been shifted by $T / 2$ with respect to Eq. (2). 
$\Omega_{\max } \simeq 56 \omega_{0}$, in good agreement with the numerical results. The only noticeable difference is a slight increase of the harmonic yield in the H50-H55 interval for the down-chirped pulse and in the H12-H30 interval for the up-chirped one. In the atomic HHG, cutoff extensions can be due to the spectral widening of chirped pulses, yet this is barely appreciable in Fig. 1(a).

This scenario changes dramatically when the pulse duration is increased. In the 10-cycle case [Fig. 1(b)], one can see that a down-chirped pulse leads to an enhancement of the harmonic yield in the $\mathrm{H} 45-\mathrm{H} 70$ region by more than 3 orders of magnitude, thus extending the cutoff energy by more than $25 \omega_{0}(\sim 40 \mathrm{eV})$. In contrast, the only visible consequence of using an up-chirped pulse is a modest increase of harmonic emission at lower frequencies (H10-H30). The effects of using a down-chirp pulse are even more pronounced in the 15-cycle case: The harmonic yield increases by almost 5 orders of magnitude in the interval $\mathrm{H} 55-\mathrm{H} 75$, thus leading to a further increase of the cutoff. As in the previous case, the use of an up-chirped pulse does not have any significant effect.

To understand the origin of the observed effects and, in particular, the role of the nuclear motion, we have plotted in Figs. 1(d)-1(f) the mean internuclear distance $\langle R\rangle$, as a function of time for all the pulses considered. As can be seen, for the 5-cycle pulse, $\langle R\rangle$ can increase up to $\sim 0.3$ a.u. with respect to the equilibrium value, while for the 10- and 15 -cycle pulses it increases up to $\sim 0.9$ a.u. and $\sim 2.0$ a.u., respectively. In particular, for the 15-cycle pulses, the molecule has enough time to dissociate before the pulse ends. These numbers barely depend on the chirp sign. This implies the following scenario (see Fig. 2). During the first pulse cycles, when the maximum intensity has not yet been

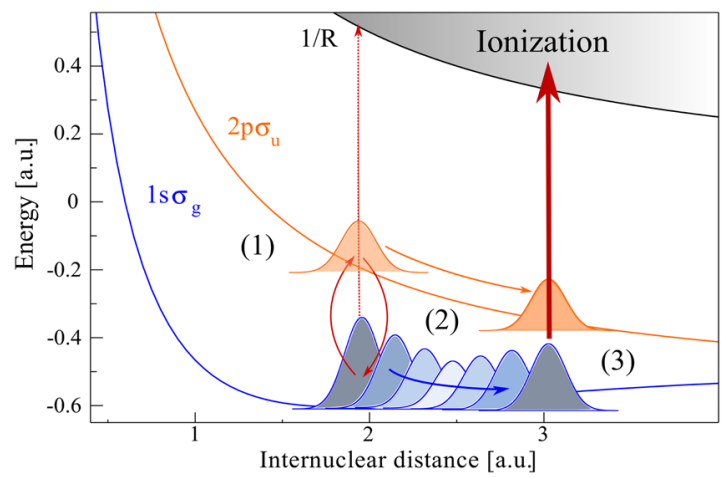

FIG. 2. Mechanism of HHG enhancement. The laser couples the $1 s \sigma_{g}$ and $2 p \sigma_{u}$ states of $\mathrm{H}_{2}^{+}$in the $\mathrm{FC}$ region, thus leading to Rabi oscillations between those states and to a coherent superposition of vibrational states (1). The ensuing nuclear wave packet then moves towards larger internuclear distances (2), where harmonic emission is more efficient than in the $\mathrm{FC}$ region due to the smaller $I_{P}$ (3). Color lines represent the potential energy curves for the lowest bound electronic states of $\mathrm{H}_{2}^{+}$; the ionization threshold is denoted by a black line. $I_{p}$ of $\mathrm{H}_{2}^{+}\left(\mathrm{T}_{2}^{+}\right)$at the equilibrium distance is 1.123 (1.133) a.u.. reached, a nuclear wave packet is created as a result of Rabi oscillations between the $1 s \sigma_{g}$ and $2 p \sigma_{u}$ states. Then this wave packet moves to larger internuclear distances, where ionization is easier due to the smaller ionization potential. As a consequence, if the pulse is long enough, so that photon absorption can effectively occur at long internuclear distances within the last few cycles, the ionization rate [51] and hence the harmonic emission [52-54] will substantially increase. The effect will be more pronounced when the laser pulse induces dissociation [Fig. 1(f)]. Now the question is: Why is such an increase in the harmonic yield observed only for down-chirped pulses?

To answer this question, we have evaluated, for the 10cycle pulses, the time-window Fourier transform (or Gabor profiles) of the dipole moment, as well as the nuclear and electronic probability densities vs time (Fig. 3). The time evolution of the nuclear probability density is similar for both up and down chirps [Figs. 3(b) and 3(e)]: It exhibits a maximum at $R>2.5$ a.u. for $t>T / 2$ and a dissociative component corresponding to the evolution of the nuclear wave packet in the excited states of the molecule. The dissociative component is more noticeable when the pulse is up-chirped, since the high-frequency cycles come at the trailing edge of the pulse, thus reducing the number of photons required to excite or ionize the molecule. As can be seen in the time-frequency distributions for the down-chirp pulse [Fig. 3(d)], the cutoff extension is entirely due to the last three harmonic bursts $(t \in[15,20] \mathrm{fs})$, which occur at the larger $R$ and correspond to the longer wavelengths. The

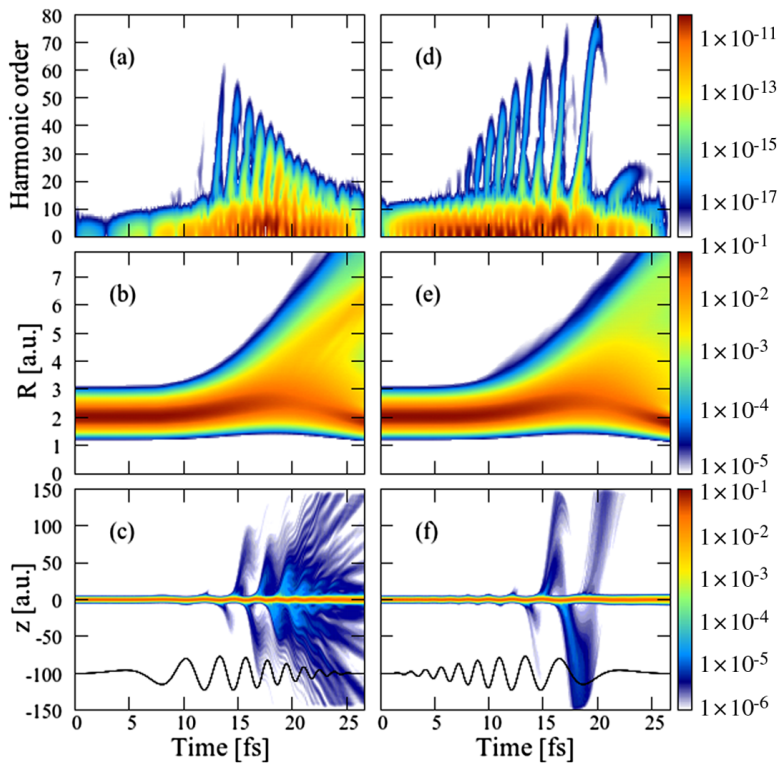

FIG. 3. (Upper row) Gabor profiles of HHG spectra from Fig. 1(b), (middle row) time evolution of the nuclear probability density, and (bottom row) time evolution of the electron probability density along the $z$ coordinate, for chirped pulses with $\alpha= \pm 1.35 \times 10^{-4}$ a.u. $E(t)$ is shown with a black line (not scaled). All plots are in the logarithmic scale. 
reverse occurs for the up-chirped pulse [Fig. 3(a)]: The longer wavelengths arrive with the early cycles, i.e., when the nuclei are still in the FC region. The plots of the electronic probability densities [Figs. 3(c) and 3(f)] show that ionization is negligible for $t<T / 2$ irrespective of the chirp. This is due to the relatively large number of photons that are needed to ionize the molecule in the $\mathrm{FC}$ region. Most ionization occurs after having reached the peak intensity, since it is in this region where $R$ is large and therefore less photons are required to ionize the molecule. Hence, as electrons driven by the low-frequency cycles acquire higher kinetic energies, i.e., are associated with higher harmonic orders, a significant cutoff extension is possible only for down-chirped pulses. This effect is reinforced by the fact that, on the second half of the pulse, the intensity decreases during the optical cycle, so that, on its way back, the electron is less decelerated than on the first half of the pulse. In general, the longer the downchirped pulse, the larger the cutoff extension, since ionization occurs at even larger $R$. There is, however, an obvious limit to the cutoff extension: The long wavelengths must be operative when the population in the ground state is still significant.

The above discussion shows that it is the adequate timing of the low-frequency cycles with respect to the nuclear wave packet dynamics that allows one to both extend the cutoff and increase the harmonic yield. An alternative way to modify this timing, which does not require varying the pulse duration, is to perform an isotopic substitution. As an illustration, Fig. 4(a) shows the harmonic spectra of $\mathrm{H}_{2}^{+}$and $\mathrm{T}_{2}^{+}$for 15-cycle up- and down-chirped pulses. As can be seen, the harmonic yield resulting from a down-chirped pulse is more than 4 orders of magnitude larger for $\mathrm{H}_{2}^{+}$than for $\mathrm{T}_{2}^{+}$. The effect is barely appreciable with up-chirped pulses. At first sight, this finding may seem counterintuitive, since at a given emission time the nuclear autocorrelation function is smaller for $\mathrm{H}_{2}^{+}$than for $\mathrm{T}_{2}^{+}$: Previous work with unchirped pulses has shown that, under this circumstance, the relative harmonic yield in the cutoff region should be the opposite to that observed here [42]. This is not the case because, in $\mathrm{T}_{2}^{+}$, the larger mass slows down the nuclear motion [see Fig. 4(b)] so much that the longer wavelengths come into play when the nuclei are still in the
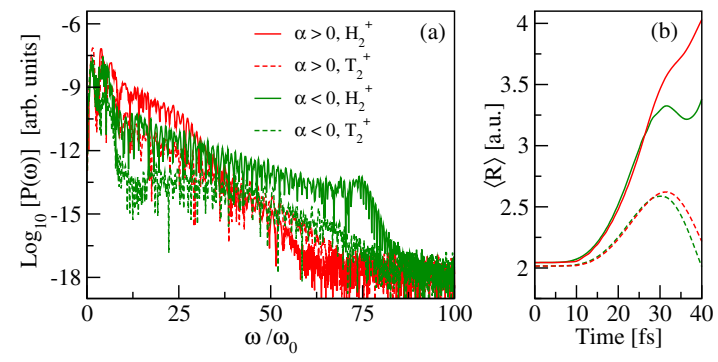

FIG. 4. (a) $\mathrm{HHG}$ spectra of $\mathrm{H}_{2}^{+}$and $\mathrm{T}_{2}^{+}$for chirped pulses with $\alpha= \pm 1.35 \times 10^{-4}$ a.u. (b) Mean value of the internuclear distance as a function of time for $\mathrm{H}_{2}^{+}$(solid lines) and $\mathrm{T}_{2}^{+}$ (dashed lines). vicinity of the $\mathrm{FC}$ region, where ionization rates are small. A similar argument explains why the difference between up- and down-chirped harmonic spectra in $\mathrm{T}_{2}^{+}$is much smaller than in $\mathrm{H}_{2}^{+}$, and it is even smaller in the infinitemass limit. For intermediate masses, one expects that the extension of the cutoff lies in between that of $\mathrm{H}_{2}^{+}$and $\mathrm{T}_{2}^{+}$. Therefore, by performing different isotopic substitutions one can control the extension of the harmonic plateau while keeping the pulse duration constant. For heavier molecules, a similar control can be achieved either by using longer pulses [55], since in this way the nuclear wave packet has enough time to reach the region of long $R$ where ionization is efficiently produced, or by increasing the laser intensity, since this favors ionization at short $R$.

In conclusion, we have shown that, as a result of subtle electron-nuclei correlation effects, the $\mathrm{HHG}$ spectrum of $\mathrm{H}_{2}^{+}$ arising from down-chirped pulses is more intense and extends to much larger harmonic orders than that resulting from unchirped or up-chirped pulses. This is the consequence of the laser-induced nuclear dynamics that drives the system to large internuclear distances, where the trailing longwavelength cycles can efficiently ionize the molecule. By varying the pulse duration or the mass of the nuclei, one can tune the time at which ionization by the long wavelengths occurs and thus control the extension of the harmonic cutoff. Similar effects are expected in other molecules.

Introducing a down chirp and eventually rescaling the laser intensity is rather straightforward by using nowadays ultrafast laser sources. For example, up and down chirps comparable to those considered in the present work can be produced by using dielectric mirrors [56] or pulse stretchers or compressors such as those used in standard chirpedpulse amplification schemes [57]. Also, due to the low ionization rates $(<1 \%$ for a 10 -cycle pulse), one can reasonably expect that potential phase mismatch problems can be easily overcome by balancing geometrical dispersion with material and plasma dispersion, e.g., with hollow waveguides or shallow-focus geometries (see, e.g., [58]). We thus believe that the general mechanism presented in this work provides an experimentally feasible approach to efficiently extend HHG spectra of light diatomic molecules (e.g., $\mathrm{H}_{2}$ ) to higher orders.

We gratefully acknowledge fruitful discussions with Y. Mairesse. This work has been accomplished with a generous allocation of computer time from Mare Nostrum BSC and CCC-UAM and has been partially supported by the European Research Council Advanced Grant No. XCHEM 290853, MINECO Project No. FIS201342002-R, ERA-Chemistry Project No. PIM2010EEC00751, European Grant No. MC-ITN CORINF, European COST Action XLIC CM1204, and the CAM project NANOFRONTMAG. R.E. F. S. acknowledges FCTFundação para a Ciência e Tecnologia, Portugal, Grant No. SFRH/BD/84053/2012. 
* Corresponding author. fernando.martin@uam.es

[1] A. McPherson, G. Gibson, H. Jara, U. Johann, T. S. Luk, I. McIntyre, K. Boyer, and C. K. Rhodes, J. Opt. Soc. Am. B 4, 595 (1987).

[2] M. Ferray, A. L'Huillier, X. Li, L. Lompre, G. Mainfray, and C. Manus, J. Phys. B 21, L31 (1988).

[3] A. L'Huillier and P. Balcou, Phys. Rev. Lett. 70, 774 (1993).

[4] P. B. Corkum, Phys. Rev. Lett. 71, 1994 (1993).

[5] M. Lewenstein, P. Balcou, M. Y. Ivanov, A. L'Huillier, and P. B. Corkum, Phys. Rev. A 49, 2117 (1994).

[6] K. J. Schafer, B. Yang, L. F. DiMauro, and K. C. Kulander, Phys. Rev. Lett. 70, 1599 (1993).

[7] P. Agostini and L. F. DiMauro, Rep. Prog. Phys. 67, 813 (2004).

[8] F. Krausz and M. Ivanov, Rev. Mod. Phys. 81, 163 (2009).

[9] M. Drescher, M. Hentschel, R. Kienberger, M. Uiberacker, V. Yakovlev, A. Scrinzi, T. Westerwalbesloh, U. Kleineberg, U. Heinzmann, and F. Krausz, Nature (London) 419, 803 (2002).

[10] M. Uiberacker et al., Nature (London) 446, 627 (2007).

[11] J. Mauritsson et al., Phys. Rev. Lett. 105, 053001 (2010).

[12] E. Goulielmakis et al., Nature (London) 466, 739 (2010).

[13] H. Wang, M. Chini, S. Chen, C.-H. Zhang, F. He, Y. Cheng, Y. Wu, U. Thumm, and Z. Chang, Phys. Rev. Lett. 105, 143002 (2010).

[14] C. Ott et al., Nature (London) 516, 374 (2014).

[15] H. Niikura, F. Légaré, R. Hasbani, M. Y. Ivanov, D. Villeneuve, and P. Corkum, Nature (London) 421, 826 (2003).

[16] S. Baker, J. S. Robinson, C. Haworth, H. Teng, R. Smith, C. Chirilă, M. Lein, J. Tisch, and J. Marangos, Science 312, 424 (2006).

[17] F. Calegari et al., Science 346, 336 (2014).

[18] M. Negro et al., Laser Phys. Lett. 8, 875 (2011).

[19] B. Schütte, P. Weber, K. Kovács, E. Balogh, B. Major, V. Tosa, S. Han, M. J. J. Vrakking, K. Varjú, and A. Rouzée, Opt. Express 23, 33947 (2015).

[20] K. Kovács, V. Tosa, B. Major, E. Balogh, and K. Varjú, IEEE J. Sel. Top. Quantum Electron. 21, 1 (2015).

[21] T. Popmintchev et al., Science 336, 1287 (2012).

[22] B. Shan and Z. Chang, Phys. Rev. A 65, 011804 (2001).

[23] E. Seres, J. Seres, F. Krausz, and C. Spielmann, Phys. Rev. Lett. 92, 163002 (2004).

[24] J. Seres, E. Seres, A. J. Verhoef, G. Tempea, C. Streli, P. Wobrauschek, V. Yakovlev, A. Scrinzi, C. Spielmann, and F. Krausz, Nature (London) 433, 596 (2005).

[25] P. Moreno, L. Plaja, V. Malyshev, and L. Roso, Phys. Rev. A 51, 4746 (1995).

[26] V. Strelkov, A. Sterjantov, N. Y. Shubin, and V. Platonenko, J. Phys. B 39, 577 (2006).

[27] K. Schiessl, K. L. Ishikawa, E. Persson, and J. Burgdörfer, Phys. Rev. Lett. 99, 253903 (2007).

[28] J. Tate, T. Auguste, H. G. Muller, P. Salieres, P. Agostini, and L. F. DiMauro, Phys. Rev. Lett. 98, 013901 (2007).

[29] J. Pérez-Hernández, D. Hoffmann, A. Zaïr, L. Chipperfield, L. Plaja, C. Ruiz, J. Marangos, and L. Roso, J. Phys. B 42, 134004 (2009).

[30] I. J. Kim, C. M. Kim, H. T. Kim, G. H. Lee, Y. S. Lee, J. Y. Park, D. J. Cho, and C. H. Nam, Phys. Rev. Lett. 94, 243901 (2005).
[31] L. E. Chipperfield, J. S. Robinson, J. W. G. Tisch, and J. P. Marangos, Phys. Rev. Lett. 102, 063003 (2009).

[32] S. Kim, J. Jin, Y.-J. Kim, I.-Y. Park, Y. Kim, and S.-W. Kim, Nature (London) 453, 757 (2008).

[33] M. F. Ciappina, J. Biegert, R. Quidant, and M. Lewenstein, Phys. Rev. A 85, 033828 (2012).

[34] L. Feng, Phys. Rev. A 92, 053832 (2015).

[35] J. Leeuwenburgh, B. Cooper, V. Averbukh, J. P. Marangos, and M. Ivanov, Phys. Rev. Lett. 111, 123002 (2013).

[36] J. J. Carrera and S.-I. Chu, Phys. Rev. A 75, 033807 (2007).

[37] W. Jie, Z. Zhen, and L. Xue-Shen, Chin. Phys. B 19, 093201 (2010).

[38] K. Kovács, E. Balogh, J. Hebling, V. Toşa, and K. Varjú, Phys. Rev. Lett. 108, 193903 (2012).

[39] L. Feng and T. Chu, J. Chem. Phys. 136, 054102 (2012).

[40] E. V. van der Zwan and M. Lein, Phys. Rev. A 82, 033405 (2010).

[41] C. Chirilă and M. Lein, J. Mod. Opt. 53, 113 (2006).

[42] M. Lein, Phys. Rev. Lett. 94, 053004 (2005).

[43] X.-B. Bian and A. D. Bandrauk, Phys. Rev. Lett. 113, 193901 (2014).

[44] F. Morales, P. Rivière, M. Richter, A. Gubaydullin, M. Ivanov, O. Smirnova, and F. Martín, J. Phys. B 47, 204015 (2014).

[45] R. E. F. Silva, P. Rivière, F. Morales, O. Smirnova, M. Ivanov, and F. Martín (to be published).

[46] P. Moreno, L. Plaja, andL. Roso, Phys. Rev. A 55, R1593 (1997).

[47] T. Zuo, S. Chelkowski, and A. D. Bandrauk, Phys. Rev. A 48, 3837 (1993).

[48] Predictions based on the solution the TDSE are basically limited to effective one-electron molecules as those considered in the present work. For complex molecules, one must rely on approximate methods such as, e.g., the semiclassical three-step model combined with the strong-field approximation [5], but these methods are still awaiting improvements to fully account for the correlated electronic and nuclear motions.

[49] T. Niederhausen, U. Thumm, and F. Martín, J. Phys. B 45, 105602 (2012).

[50] M. Wollenhaupt, T. Bayer, and T. Baumert, in Ultrafast Dynamics Driven by Intense Light Pulses. From Atoms to Solids, from Lasers to Intense X-Rays, edited by M. Kitzler and S. Gräfe (Springer, New York, 2016), pp. 63-122.

[51] V. S. Popov, Phys. Usp. 47, 855 (2004).

[52] S. Chelkowski, C. Foisy, and A. D. Bandrauk, Phys. Rev. A 57, 1176 (1998).

[53] A. D. Bandrauk, S. Chelkowski, S. Kawai, and H. Lu, Phys. Rev. Lett. 101, 153901 (2008).

[54] T. Pfeifer, D. Walter, G. Gerber, M. Y. Emelin, M. Y. Ryabikin, M. D. Chernobrovtseva, and A. M. Sergeev, Phys. Rev. A 70, 013805 (2004).

[55] M. Lara-Astiaso, R. E. F. Silva, C. Meier, and F. Martín (to be published).

[56] F. X. Kärtner, U. Morgner, R. Ell, T. Schibli, J. G. Fujimoto, E. P. Ippen, V. Scheuer, G. Angelow, and T. Tschudi, J. Opt. Soc. Am. B 18, 882 (2001).

[57] K.-H. Hong, J. H. Sung, Y. S. Lee, and C. H. Nam, Opt. Commun. 213, 193 (2002).

[58] O. Cohen, X. Zhang, A. L. Lytle, T. Popmintchev, M. M. Murnane, and H. C. Kapteyn, Phys. Rev. Lett. 99, 053902 (2007). 